HEFAT2012

$9^{\text {th }}$ International Conference on Heat Transfer, Fluid Mechanics and Thermodynamics

16 - 18 July 2012

Malta

\title{
NUMERICAL MODELING OF ASPHALTENE DEPOSITION
}

\author{
Q. Ge, ${ }^{1}$ Y.F. Yap, ${ }^{1}$ F.M. Vargas, ${ }^{2}$ M. Zhang, ${ }^{3}$ J.C. Chai ${ }^{1 *}$ \\ *Author for correspondence \\ ${ }^{1}$ Department of Mechanical Engineering, ${ }^{2}$ Department of Chemical Engineering, \\ The Petroleum Institute, \\ Abu Dhabi, UAE. \\ ${ }^{3}$ School of Energy and Power Engineering, Nanjing University of Science and Technology, Nanjing, P.R. China. \\ E-mail: jchai@pi.ac.ae
}

\begin{abstract}
Asphaltene deposition on the walls of wellbore or pipeline during oil production and processing will cause dire consequences if left untreated. A better understanding of this phenomenon is required. This study presents a general modelling framework based either on the level-set approach or the concentration approach for modelling asphaltene deposition processes. Both the level-set method and the total concentration approach belong to the family of front capturing methods. Therefore, the dynamics of the depositing front can be captured implicitly on fixed mesh. The presented frameworks are validated against each other for general deposition process. Preliminary parametric studies are then performed to study the influences of various factors on the deposition process.
\end{abstract}

\section{INTRODUCTION}

Figure 1 shows an oil reservoir connected to a wellbore. Crude oil contains various heavy organics such as asphaltene. Asphaltene is the heavy fraction of crude oil which is soluble in some species like aromatics but insoluble in others [1]. At reservoir condition, dissolved asphaltene is stable. During oil production, dissolved asphaltene is carried by the flowing oil along the wellbore. There is a drop in both pressure and temperature along the wellbore. At a certain location of the wellbore, dissolved asphaltene becomes unstable and precipitates out of the oil in the form of particles. Some of these particles will be carried by the oil downstream but some would stick and deposit onto the wall of the wellbore, forming a layer of asphaltene deposit. The formation of asphaltene deposit on the wall of the wellbore reduces the cross sectional area for oil to flow and increases pressure drop. If left untreated, the whole wellbore can be blocked.

The problem of asphaltene deposition in the oil/gas industry has been investigated for more than three decades. The understandings gained though substantial, is still far from being satisfactory. Modeling of asphaltene deposition in well-bore or pipeline is currently still being actively pursued.
Modeling of asphaltene deposition requires two essential components. These are (1) thermodynamic model and (2) transport model. Thermodynamic model serves to provide information on precipitation of dissolved asphaltene. This was pursued for example in [2]-[5]. The transport of precipitated asphaltene particles and their subsequent deposition on surfaces are described in the transport model. Given the lacking in the understandings of the rich physics involved in the deposition process, the transport model is frequently of a mechanistic nature. It relies heavily on experimental data and therefore applicable within a very limited range [6]-[10]. A more complete treatment of the transport model requires a computational fluid dynamic approach (CFD) and this is however not pursued actively in the existing literatures. The model of [11] and its one-dimensional version of [12] have strong flavour of CFD, although fluid flow modeling is not considered.

The evolving depositing front is one of the most important features in modeling of asphaltene deposition. It dictates the structure and thickness of the layer of asphaltene deposit. Common to most existing transport models for asphaltene deposition is the assumption of a static depositing front, i.e. the depositing front does not evolve during the deposition process. Such an assumption is understandable given the complications arise with an evolving depositing front, leading to a moving boundary problem. This assumption is generally not appropriate as the deposited asphaltene layer is not thin compared to the size of the well-bore or pipeline. A more accurate transport model therefore requires such an assumption be removed. This leads nicely to the aim of the present study. This study presents a general framework of modelling asphaltene deposition process. It focuses at the CFD transport model and emphasizes on the evolving depositing front that is strongly coupled to the associated fluid, mass and energy transport. The present study, however, does not aim to delve deeply into the rich physics involved in asphaltene deposition 
processes but rather to provide a means to facilitate the investigation of such phenomena.

The remaining article is divided into 6 sections. The problem description is given in Section 2. A general CFD framework is described in Section 3. This is followed by the mathematical formulation in Section 4. The solution procedure is then briefly outlined in Section 5. In Section 6, the results are presented and discussed. Finally, the article concludes with a few remarks.

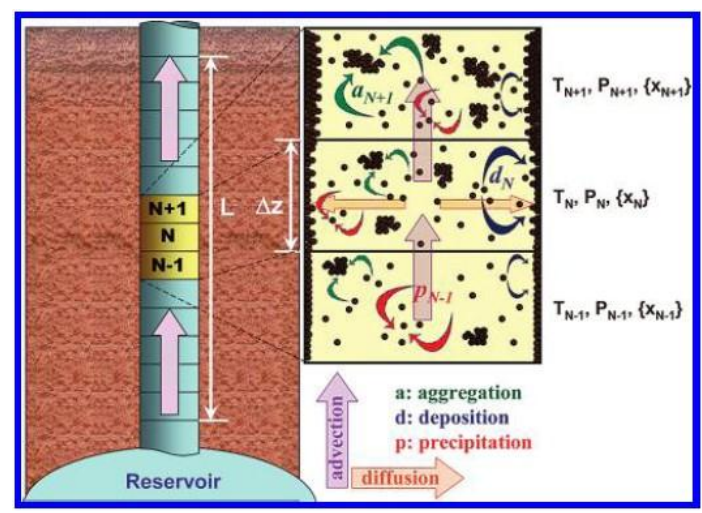

Figure 1: Oil flowing from an oil reservoir into a production well [11].

\section{NOMENCLATURE}

C particle concentration

$C_{D} \quad$ concentration of particles in the form of deposit

$C_{o}$ the concentration of asphaltene in solution at inlet condition

$C_{T} \quad$ total concentration

$D$ diffusion coefficient

$D a_{D}$ Damkoler number

$k_{D} \quad$ reaction rate for deposition

$\hat{n}_{i} \quad$ unit normal vector at the depositing front

$p \quad$ pressure

$P e \quad$ Peclet number

$\vec{q} \quad$ deposition flux

Re Reynolds number

$S \quad$ source/sink of the particles

$t$ time

$\vec{u}_{i} \quad$ velocity of the depositing front

$\vec{u}_{i, \text { ext }}$ velocity extended from $\vec{u}_{i}$

$\vec{x} \quad$ position vector

$\delta \quad$ modified Dirac delta function

$\phi \quad$ level-set function

$\mu \quad$ viscosity of fluid

$\rho$ density of fluid

$\rho_{D} \quad$ density of the particle deposit

$\Gamma \quad$ depositing front

$\Omega \quad$ domain of interest

$\Omega_{+} \quad$ fluid region

$\Omega_{-} \quad$ deposit region
Superscript

* dimensionless quantity

\section{PROBLEM DESCRIPTION}

From a modeling point of view, an asphaltene deposition problem in a wellbore can be view as follows. Shown in Fig. 2 is a schematic of the domain of interest $\Omega$, i.e. a wellbore with an inflow and an outflow. It consists of a fluid region $\Omega_{F}$ (oil) and a deposit region $\Omega_{D}$ (asphaltene deposit), i.e. $\Omega=\Omega_{F}(t) \cup \Omega_{D}(t)$. These two regions are separated by the depositing front $\Gamma(t)$. Oil flows into the wellbore at the inlet. It carries both dissolved asphaltene and precipitated asphaltene particles. Additional asphaltene particles can precipitate along the wellbore. In the wellbore, asphaltene particles aggregate to form larger particles. These particles gradually deposit onto the depositing front. As a result of the deposition, the depositing front evolves with a velocity of $\vec{u}_{i}$ and the deposit region formed by asphaltene particles grows. After $\Delta t$, additional layer of asphaltene particle deposit forms.

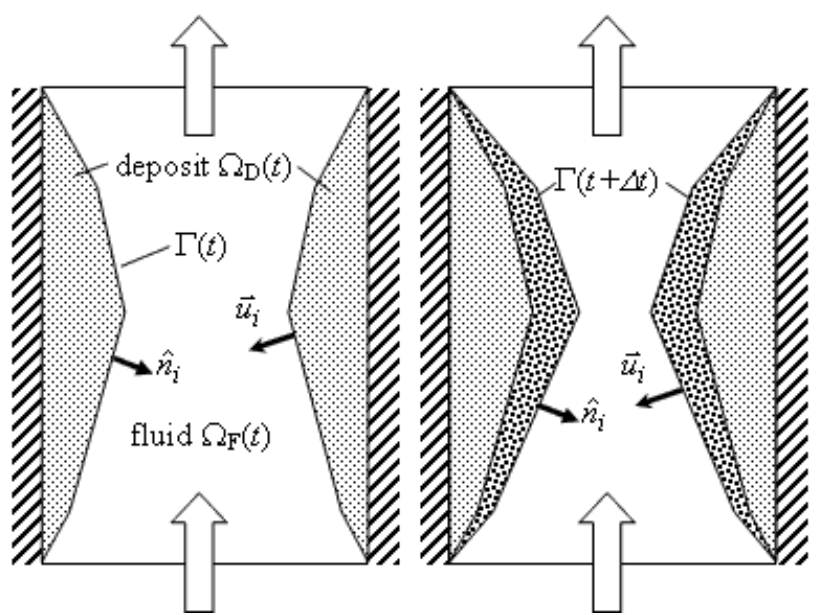

Figure 2: Mathematical modeling of an asphaltene deposition process.

\section{GENERAL MODELING FRAMEWORK}

Depicted in Figure 3 is a general framework of an asphaltene deposition model. It consists of two components: (1) the thermodynamic model and (2) the CFD model. The thermodynamic model determines the condition at which dissolved asphaltene becomes unstable and precipitates out of oil to form asphaltene particles. The CFD model on the other hand describes if these asphaltene particles are deposited on the wall of the well or just carried by the flowing oil downstream.

The focus of the current work is on the CFD model. The CFD model needs to have the following features. Since the asphaltene particles are transported in the well, a particle transport model is required to describe the temporal and spatial distribution of the asphaltene particles. Such a transport process is governed by species conservation equation. One of major transport mechanisms of these asphaltene particles is of course convection by the flowing oil. The flow of oil can be modeled using the Navier-Stokes equations. If needed, non-Newtonian 
behavior of oil should be incorporated accordingly. Some of the asphaltene particles will stick and deposit onto the wall of the wellbore initially and at a later stage onto the depositing front. This deposition process at the depositing front needs to be modeled. Analogy with surface chemical reaction can be made use of. The deposition of asphaltene particles onto the depositing front can be modeled as a 1st order reaction. Once deposition occurs, the deposit layer grows and depositing front moves. This dynamically evolving depositing front needs to be tracked or captured. Here in this article, the depositing front is captured via two different methods: (1) The level-set method [13] and (2) the total concentration method [14]. The total concentration method for deposition process was recently developed. Both of these methods are of the front-tracking family, as such a fixed mesh can be employed for numerical solution. The asphaltene deposit can be modeled as an impermeable solid.

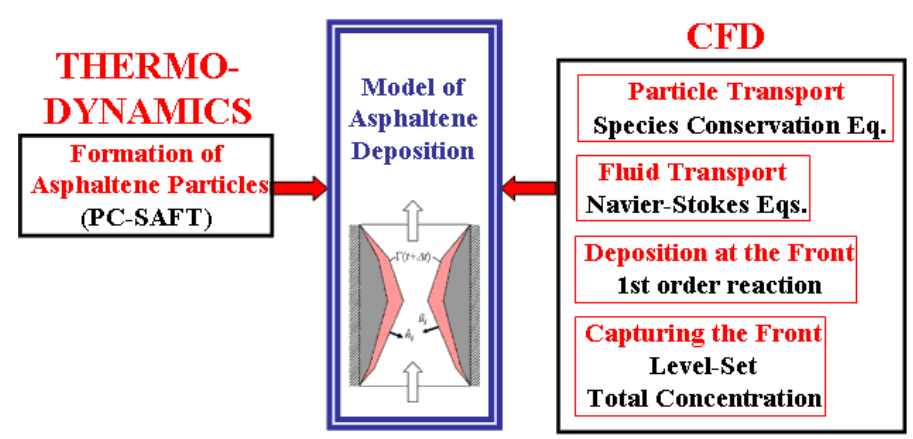

Figure 3: Model of an asphaltene deposition process.

\section{MATHEMATICAL FORMULATION}

In the current form of the model, the thermodynamics model has yet been incorporated into the model. In the following sections, the governing equations for the CFD model will be given. We delay presenting the species conservation equation to the end of this section, as the form of this equation is intimately related to the front capturing approach adopted.

\section{Models for Fluid Transport}

The particles are carried by a flowing fluid. Fluid flow is modeled using the continuity and the Navier-Stokes equations for the whole domain $\Omega$.

$$
\begin{aligned}
& \frac{\partial \rho}{\partial t}+\nabla \bullet(\rho \vec{u})=0, \forall \vec{x} \in \Omega \\
& \frac{\partial(\rho \vec{u})}{\partial t}+\nabla \bullet(\rho \vec{u} \vec{u})=-\nabla p+\nabla \bullet\left[\mu\left(\nabla \vec{u}+\nabla \vec{u}^{T}\right)\right], \forall \vec{x} \in \Omega
\end{aligned}
$$

where $\rho$ and $\mu$ are the fluid density and viscosity respectively. For the velocity, the boundary condition can be a combination of inlet velocity, outflow boundary and no slip.

\section{Model for Deposition at the Depositing Front}

The deposition process for the asphaltene particles at the depositing front is modeled as a first order reaction with the deposition flux expressed as

$$
\vec{q}=-\rho_{D} \vec{u}_{i}=-k_{D} C \hat{n}_{i} \quad, \vec{x} \in \Gamma(t)
$$

where $\rho_{D}, k_{D}, C$ and $\hat{n}_{i}$ are the density of the deposit, the deposition reaction rate, the particle concentration and unit normal vector pointing into the fluid region respectively. If the deposition process is instead modeled as a second order reaction, Eq. (3) needs to be modified accordingly. However, the methods presented here can account for a second order reaction easily by including some minor modifications.

\section{Methods to Capture the Depositing Front}

Capturing the evolving depositing front is the main challenge of a deposition model. For this purpose, two separate methods are used. These are the level-set approach and the newly developed total concentration approach. The conservation equation governing the transport of particles is slightly different depends on the approach used in capturing the depositing front.

\section{Level-Set Approach}

In the level-set approach [13], the depositing front is represented using a level-set function defined as

$\phi \equiv \begin{cases}-d, & \text { if } \vec{x} \in \Omega_{D}(t) \\ 0, & \text { if } \vec{x} \in \Gamma(t) \\ +d, & \text { if } \vec{x} \in \Omega_{F}(t)\end{cases}$

where $d$ is the shortest distance from the depositing front. Under such a representation, the depositing front is given by $\phi=0$. The movement of the depositing front can be captured by

$$
\frac{\partial \phi}{\partial t}+\vec{u}_{i, e x t} \bullet \nabla \phi=0 \quad, \forall \vec{x} \in \Omega
$$

where $\vec{u}_{i, \text { ext }}$ is the extended velocity of the depositing front $\vec{u}_{i}$. The conservation equation governing the transport of asphaltene particles is given by

$\frac{\partial C}{\partial t}+\nabla \bullet(\vec{u} C)=\nabla \bullet(D \nabla C)+S-k_{D} C \delta(\phi)|\nabla \phi|, \forall \vec{x} \in \Omega$

where $\vec{u}, D$ and $\delta(\phi)$ are velocity of the fluid, diffusion coefficient and the Dirac delta function respectively. In Eq. (6), $S$ is the source/sink of the particles. The last term in Eq. (6) accounts for the particle consumed during the deposition process. The following initial and boundary conditions are enforced.

Initial Condition

$C(\vec{x}, 0)= \begin{cases}0, & \text { if } \vec{x} \in \Omega_{D} \\ C_{o}(\vec{x}), & \text { if } \vec{x} \in \Omega_{F}\end{cases}$

Boundary conditions

$C(\vec{x}, t)=C^{P}(\vec{x}), \quad \forall \vec{x} \in \partial \Omega^{C}, t \geq 0$

$-D \nabla C(\vec{x}, t) \bullet \hat{n}=q_{n}^{P}(\vec{x}), \quad \forall \vec{x} \in \partial \Omega^{q n}, t \geq 0$

where $\partial \Omega^{C} \cup \partial \Omega^{q n}=\partial \Omega, \partial \Omega^{C} \cap \partial \Omega^{q n}=\varnothing$ and $\partial \Omega^{C} \neq \varnothing$.

\section{Total Concentration Approach}

In the total concentration approach [14], the species conservation equation for the asphaltene particles is derived in 
terms of the total concentration $C_{T}$. It is defined as the the sum of the concentration of the to-be-deposited (particles, $C$ ) and deposited materials (deposit, $C_{D}$ ), i.e.

$C_{T}=C+C_{D}$

With this definition, the conservation equation for the particles is given by

$\frac{\partial C_{T}}{\partial t}+\nabla \bullet(\vec{u} C)=\nabla \bullet(D \nabla C)+S, \forall \vec{x} \in \Omega$

or

$\frac{\partial C}{\partial t}+\nabla \bullet(\vec{u} C)=\nabla \bullet(D \nabla C)+S-\frac{\partial C_{D}}{\partial t}, \forall \vec{x} \in \Omega$

$C_{D}$ is evaluated by incorporating the interfacial condition at the depositing front into a discretized form of Eq. (10). With this the devolving depositing front is captured implicitly by $C_{D}$. It has a value of $0 \leq C_{D} \leq \rho_{D}$ with $C_{D}=0$ for a control volume with no deposit and $C_{D}=\rho_{D}$ for a control volume with fully filled deposit. In the solution of Eq. (10), the following initial and boundary conditions apply.

Initial Condition

$C(\vec{x}, 0)= \begin{cases}0, & \text { if } \vec{x} \in \Omega_{D} \\ C_{o}(\vec{x}), & \text { if } \vec{x} \in \Omega_{F}\end{cases}$

$C_{D}(\vec{x}, 0)= \begin{cases}0, & \text { if } \vec{x} \in \Omega_{F} \\ C_{D o}(\vec{x}), & \text { if } \vec{x} \in \Omega_{D}\end{cases}$

Boundary conditions

$C(\vec{x}, t)=C^{P}(\vec{x}), \quad \forall \vec{x} \in \partial \Omega^{C}, t \geq 0$

$-D \nabla C(\vec{x}, t) \bullet \hat{n}=q_{n}^{P}(\vec{x}), \forall \vec{x} \in \partial \Omega^{q n}, t \geq 0$

where $\partial \Omega^{C} \cup \partial \Omega^{q n}=\partial \Omega, \partial \Omega^{C} \cap \partial \Omega^{q n}=\varnothing$ and $\partial \Omega^{C} \neq \varnothing$.

The boundary condition for $C_{D}$ is not required.

\section{SOLUTION PROCEDURES}

\section{Numerical Implementations}

The conservation equations (Eqs. 1, 2, 6 and 10) can be written in the form of a generic transient convection-diffusion equation. This generic equation is solved using the finite volume method [15]-[16] on a staggered mesh arrangement. Scalar variables are defined at the node of the CVs. The staggered velocity components are defined at the surface of the CVs. The combined convection-diffusion effect is modeled using the Power Law. A fully implicit scheme is used for time integration. The velocity-pressure coupling of the NavierStokes equations is handled with the SIMPLER algorithm.

The level-set approach requires higher order numerical schemes to capture the evolving front accurately. The evolution of the level-set function (Eq. 5) is spatially discretized with WENO5 [17] and integrated using TVD-RK2 [18]. These schemes are computationally intensive. To reduce the computational effort, the level-set method is implemented in a narrow-band procedure [19] where the level-set function is solved only within a band of certain thickness from the interface. This reduces one order of computational effort.
Computer programs for both the level-set approach and the total concentration approach were written on Fortran 77 platform. At this stage, the applicability of the presented approaches is of utmost importance. Therefore, implementations were made for two-dimensional Cartesian mesh.

\section{Solution Algorithms}

The overall solution procedure for the level-set approach can be summarized as follows:

(1) Specify the initial conditions (i.e. $t=0$ ) of $\vec{u}, p, \phi$ and C.

(2) Advance the time step to $t+\Delta t$.

(3) Solve Eqs. (1) and (2) for $\left.\vec{u}\right|_{t+\Delta t}$ and $\left.p\right|_{t+\Delta t}$.

(4) Solve Eq. (6) for $\left.C\right|_{t+\Delta t}$.

(5) Calculate $\left.\vec{u}_{i}\right|_{t+\Delta t}$ from Eq. (3) and then calculate $\left.\vec{u}_{i, e x t}\right|_{t+\Delta t}$.

(6) Solve Eq. (5) for $\left.\phi\right|_{t+\Delta t}$.

(7) Repeat steps (3) to (6) until the solution converges.

(8) Repeat steps (2) to (7) for all time steps.

The solution procedure for the total concentration approach is slightly different and is summarized as follows:

(1) Specify the initial conditions $(t=0)$ of $\vec{u}, p, C$ and $C_{D}$.

(2) Advance the time step to $t+\Delta t$.

(3) Solve Eqs. (1) and (2) for $\left.\vec{u}\right|_{t+\Delta t}$ and $\left.p\right|_{t+\Delta t}$.

(4) identify the depositing control volumes. These are control volumes of the fluid region adjacent to the deposit region.

(5) calculate $\left.C_{D}\right|_{t+\Delta t}$ for the depositing control volumes.

(6) if $\left.C_{D}\right|_{t+\Delta t}>1$, set $\left.C_{D}\right|_{t+\Delta t}=1$.

(7) solve for $\left.C\right|_{t+\Delta t}$ from Eq. (10).

(8) repeat steps (3) to (7) until the solution converges.

(9) repeat steps (2) to (8) for all time steps.

\section{RESULTS AND DISCUSSIONS}

Representative test cases for deposition process are given in this section. As these exercises aim to establish the validity of the implemented approaches in capturing the evolving depositing front with the fluid flow coupled, the source term in the species conservation equation is temporarily switched off, i.e. $S=0$.

For the ease of discussions, the following dimensionless parameters are used in the remainder of the article. These are the dimensionless particle concentration, Peclet, Damkohler and Reynolds numbers defined respectively as

$$
\begin{aligned}
C_{o}^{*} & =\frac{C_{o}}{\rho_{D}} \\
P e & \equiv \frac{u_{o} L}{D}
\end{aligned}
$$




$$
\begin{aligned}
& D a_{D} \equiv \frac{k_{D} L}{D} \\
& \operatorname{Re} \equiv \frac{\rho u_{o} L}{\mu}
\end{aligned}
$$

where $L$ and $u_{o}$ are the characteristic length and velocity respectively. The dimensionless time, coordinates and concentration are given respectively by

$$
\begin{aligned}
t^{*} & =\frac{u_{o} t}{L} \\
x^{*} & =\frac{x}{L} \\
y^{*} & =\frac{y}{L} \\
C^{*} & =\frac{C}{\rho_{D}}
\end{aligned}
$$

In the case when there is no fluid flow, the characteristic velocity is redefined as $u_{o}=D / L$ and therefore the Peclet number reduces to $P e=1$.

\section{Deposition in a One-Dimensional Semi-Infinite Domain}

Figure 4a shows a schematic of a deposition process in a one-dimensional semi-infinite domain. At $t^{*}=0$, the particle concentration in the fluid region is uniformly set to $C_{o}^{*}$ and the depositing front is located at $x^{*}=0$. As there is not fluid flow involved, diffusion is the sole mechanism of transporting the particles. During the deposition process, particles are deposited onto the depositing front resulting in the movement of the depositing front. For a given $t^{*}>0$, the depositing front is located at $x^{*}=\delta^{*}$ after an additional deposit layer of thickness $\delta^{*}$ formed on the existing depositing front. Since particles are consumed in the process, the particle concentration decreases. The initial and boundary conditions correspond to this problem are

Initial condition:

$$
C^{*}=C_{o}^{*} \text { for } 0 \leq x^{*} \leq \infty
$$

Boundary conditions:

$$
\begin{aligned}
& \frac{\partial C^{*}}{\partial x}=0 \text { for } x^{*}=0 \\
& C^{*}=C_{\infty}^{*} \text { for } x^{*}=\infty
\end{aligned}
$$

Figure $4 \mathrm{~b}$ shows the effect of $D a_{D}$ on the thickness of the deposit layer obtained from the level-set approach (LS) and the total concentration approach (TC). For these cases, the initial particle concentration is set to $C_{o}^{*}=0.5$. Although not shown here, these are grid independent solutions. To enforce the boundary condition of Eq. (20c), solutions were obtained for $x^{*}=5$ and 10 . These solutions are identical. Therefore, $x^{*}=5$ is sufficient numerically to represent a semi-infinite domain.
Generally, $\delta^{*}$ grows faster with a larger $D a_{D}$. The predictions of are in good agreement.
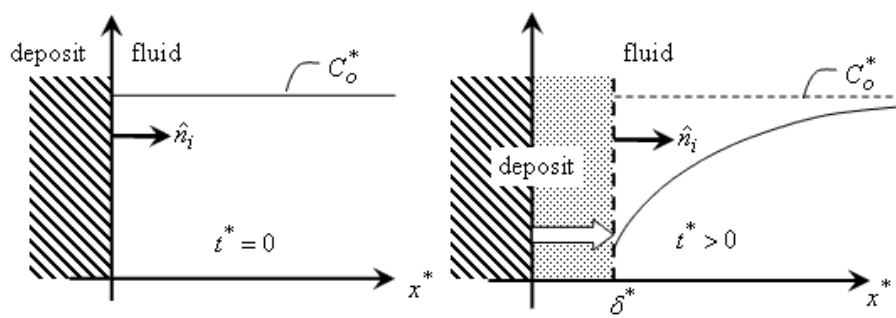

(a)

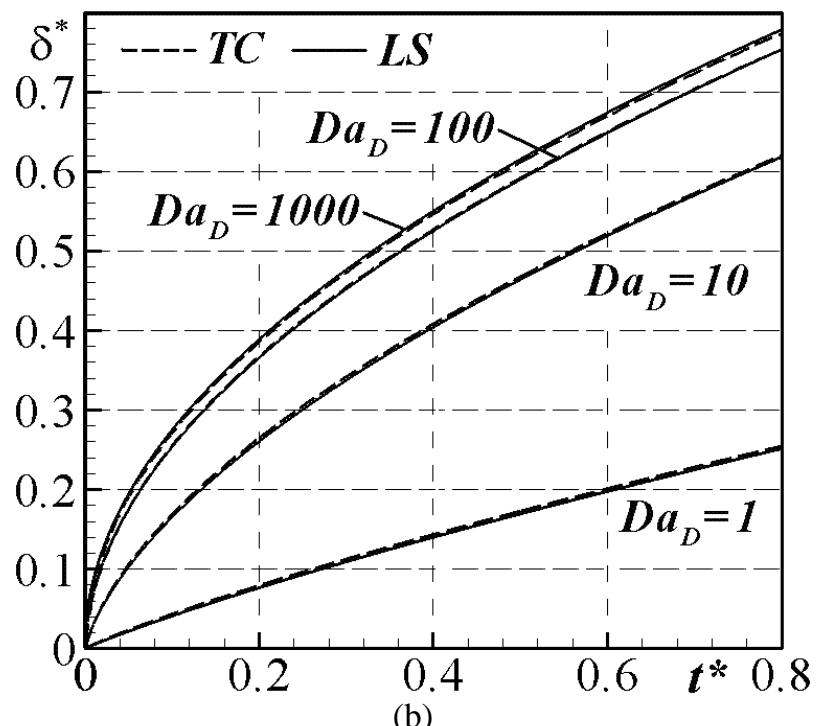

Figure 4: One-dimensional deposition, (a) domain of interest and (b) effect of $D a_{D}$ with $C_{\infty}^{*}=0.5$.

\section{Deposition in a Two-Dimensional Square Enclosure}

Figure 5a shows a two-dimensional square enclosure containing a uniform suspension of particles. Driven solely by diffusion, these particles deposit gradually on the four walls. Due to symmetry, the lower left quarter of the enclosure is modeled with the following initial and boundary conditions. Initial condition:

$C^{*}=C_{o}^{*}$ for $0 \leq x^{*} \leq 1$ and $0 \leq y^{*} \leq 1$

Boundary conditions:

$\frac{\partial C^{*}}{\partial x}=0$ for $x^{*}=0,1$

$\frac{\partial C^{*}}{\partial y}=0$ for $y^{*}=0,1$

Solutions were obtained using LS and TC approaches. Figures $5 \mathrm{~b}$ and $5 \mathrm{c}$ show the solutions for the case of $D a_{D}=1$ and 10 respectively. The predictions of the two approaches are in good agreement. Again, this comparison verifies the presented approaches. 


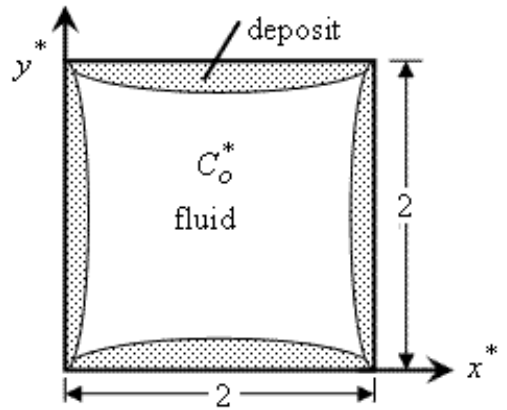

(a)

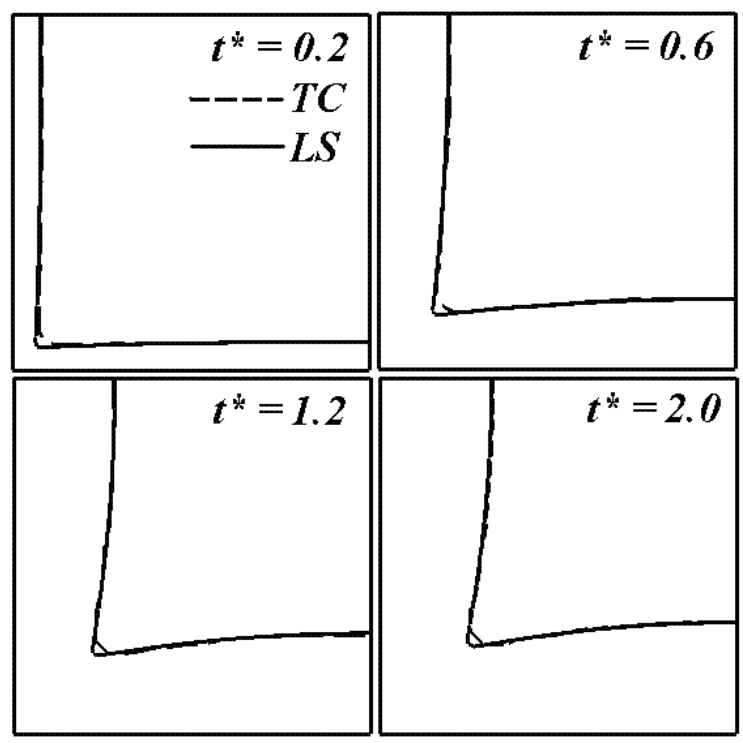

(b)

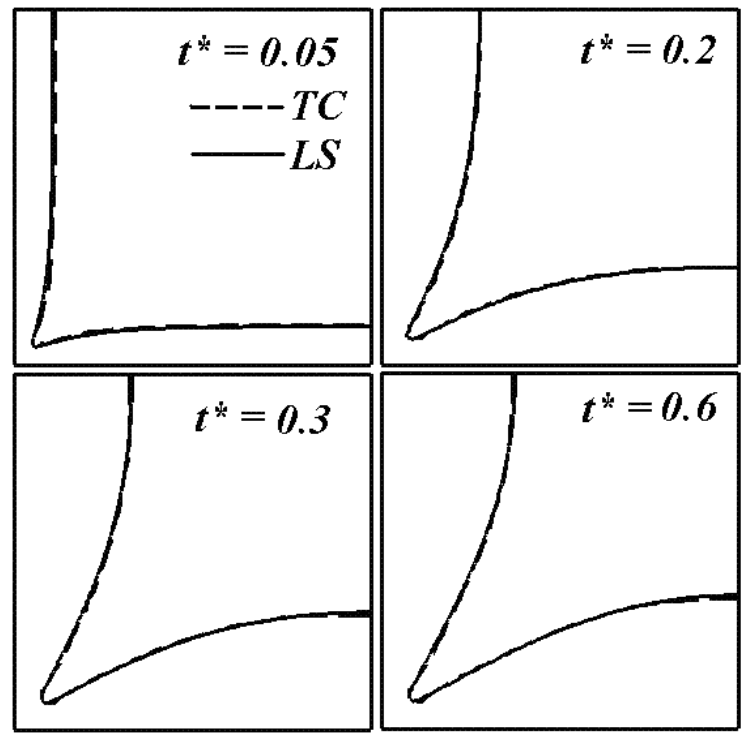

(c)

Figure 5: Deposition in a two-dimensional square enclosure with $C_{o}^{*}=0.5$, (a) domain of interest, (b) $D a_{D}=1$ and (c) $D a_{D}=10$.

\section{Deposition in Two-Dimensional Channel with Flowing Fluid}

A fluid carrying particles in the form of suspension flows into a two-dimensional channel as shown in Fig. 6a. The dimensionless length and height of the channel are 3 and 1 units. Initially, there is no deposit in the channel. As the fluid flows, the particles deposit onto the walls of the channel, forming deposit layers. Since the deposit is impermeable, it changes the flow field. The flow field and the concentration field are therefore coupled together. Making use of the symmetry of the problem, solution was computed only for the lower half of the domain. The following initial and boundary conditions apply.

Initial conditions:

$\vec{u}^{*}=\overrightarrow{0}, C^{*}=0$ for $0 \leq x^{*} \leq 3$ and $0 \leq y^{*} \leq 0.5$

Boundary conditions:

At the inlet $\left(x^{*}=0\right)$

$u^{*}=1, v^{*}=0, C^{*}=\left\{\begin{array}{cl}C_{o}^{*} & , 0.25 \leq y^{*} \leq 0.5 \\ 0 & , \text { otherwise }\end{array}\right.$

At the outlet $\left(x^{*}=3\right)$

$\frac{\partial u^{*}}{\partial x^{*}}=0, v^{*}=0, \frac{\partial C^{*}}{\partial x^{*}}=0$

At the wall $\left(y^{*}=0\right)$

$\vec{u}^{*}=\overrightarrow{0}, \frac{\partial C^{*}}{\partial y^{*}}=0$

At the symmetric plane $\left(y^{*}=0.5\right)$

$\frac{\partial u^{*}}{\partial y^{*}}=0, v^{*}=0, \frac{\partial C^{*}}{\partial y^{*}}=0$

Figure $6 \mathrm{~b}$ shows a comparison for the LS and TC approaches. The predictions of the two approaches are in good agreement. To investigate the effect of $D a_{D}$, the cases of $D a_{D}=10$ and $D a_{D}=5$ are considered. The profiles of the depositing front together with the flow field for these cases are given in Figs. 7a and 7b. Only one in every three vectors in the $x^{*}$-direction is shown to avoid overcrowding the figures. A larger $D a_{D}$ corresponds to a higher deposition reaction rate. Under such a condition, the deposit layer grows faster. Generally, the deposit layer is thickest near the inlet. This region has a much higher particle concentration. Most of the particles carried by the flow get deposited on the wall near the inlet. As the deposit layer grows, it blocks a larger cross sectional area of the channel and in effect increases the fluid velocity. The fluid velocity is in fact highest at the location of thickest deposit layer. This convects more particles to the region adjacent to this portion of the depositing front. The deposit layer grows even faster. This amplifying effect continues until the deposit layer blocks the entire channel. On the other hand, a smaller amount of particles make it to 
downstream, so the thickness of the deposit layer decreases along the flow direction.

The effect of $P e$ can be investigated by examining Figs. 7a and 7c. Upon increasing $P e$ to 20 shown Fig. 7c, very similar profile of the depositing front though with the deposit layer growing slightly slower is obtained. With a larger $P e$, instead of being deposited on the depositing front near the inlet, more particles are carried by the flow downstream due to a stronger convective transport.

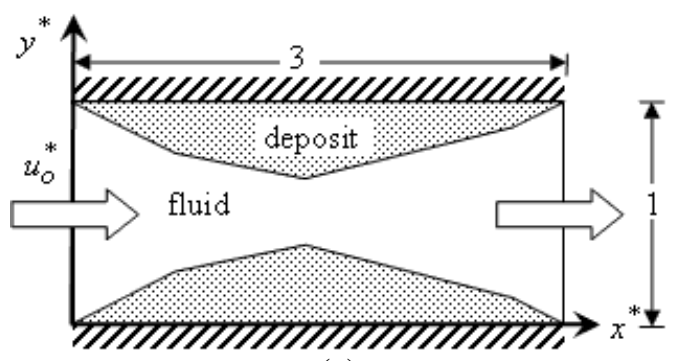

(a)

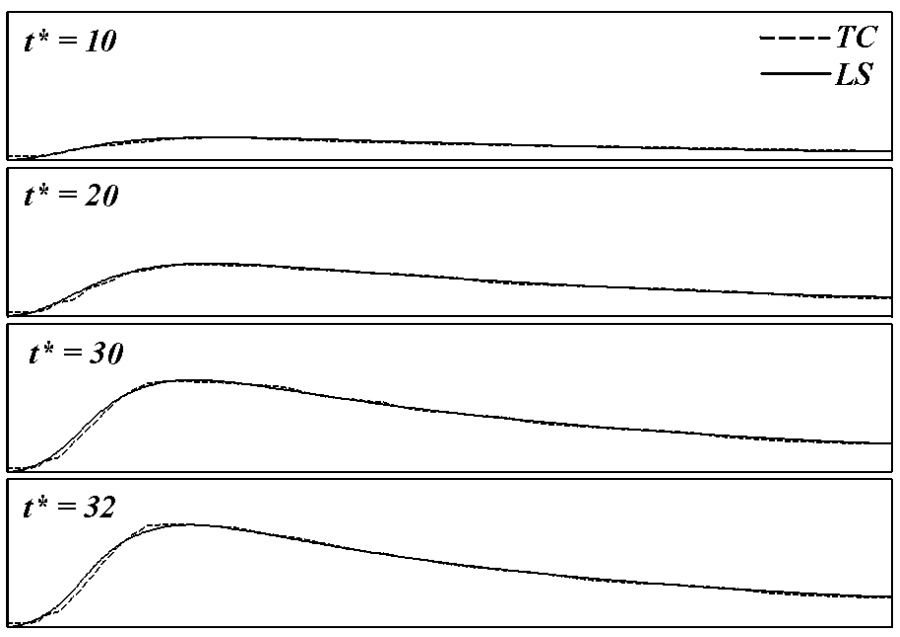

(b)

Figure 6: Deposition on the walls of a two-dimensional channel with flowing fluid, (a) domain of interest and (b) evolution of the depositing front for $\operatorname{Re}=1, C_{o}^{*}=0.1 P e=15$ and

$$
D a_{D}=10 \text {. }
$$

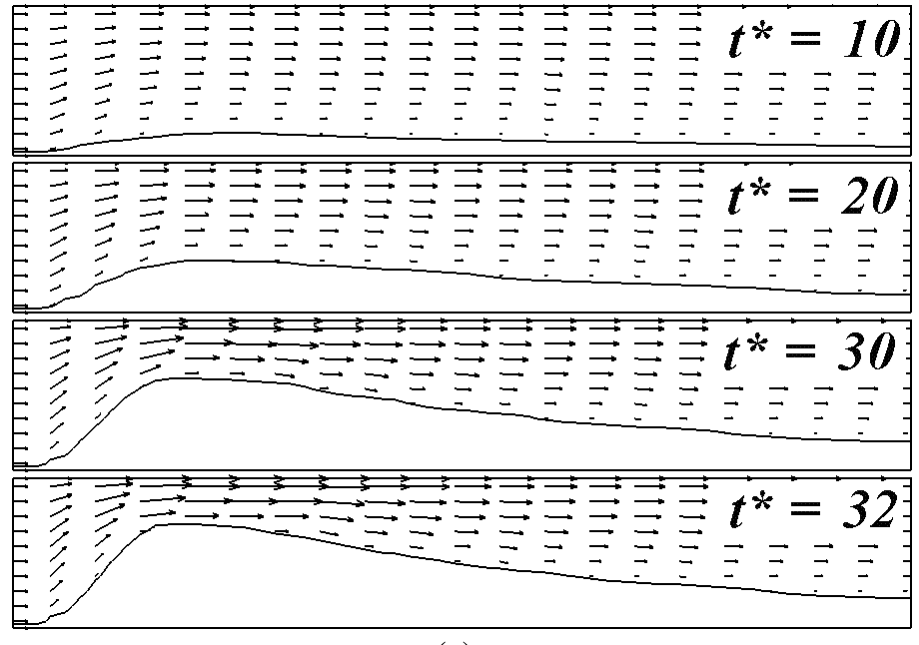

(a)

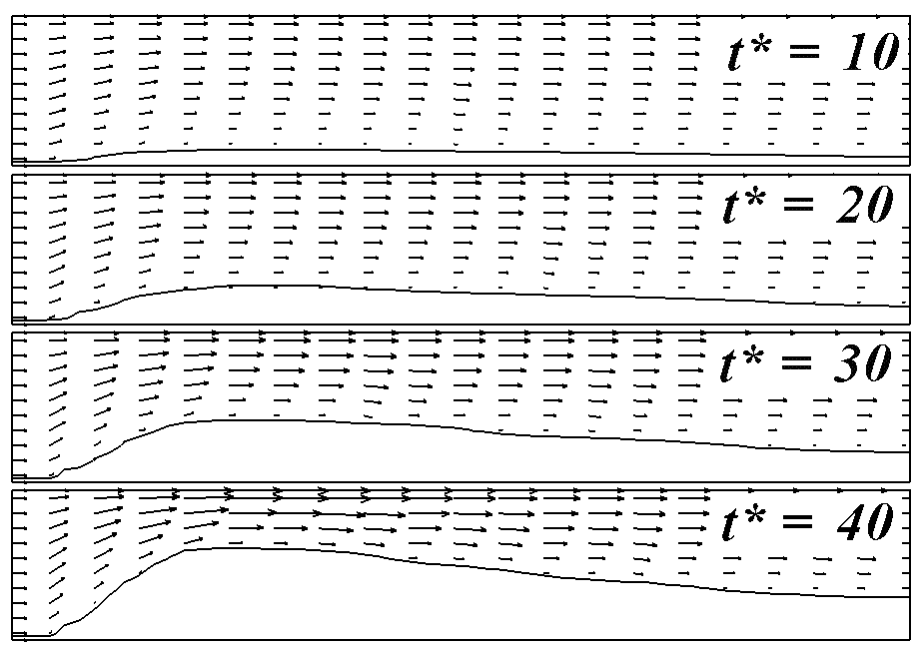

(b)

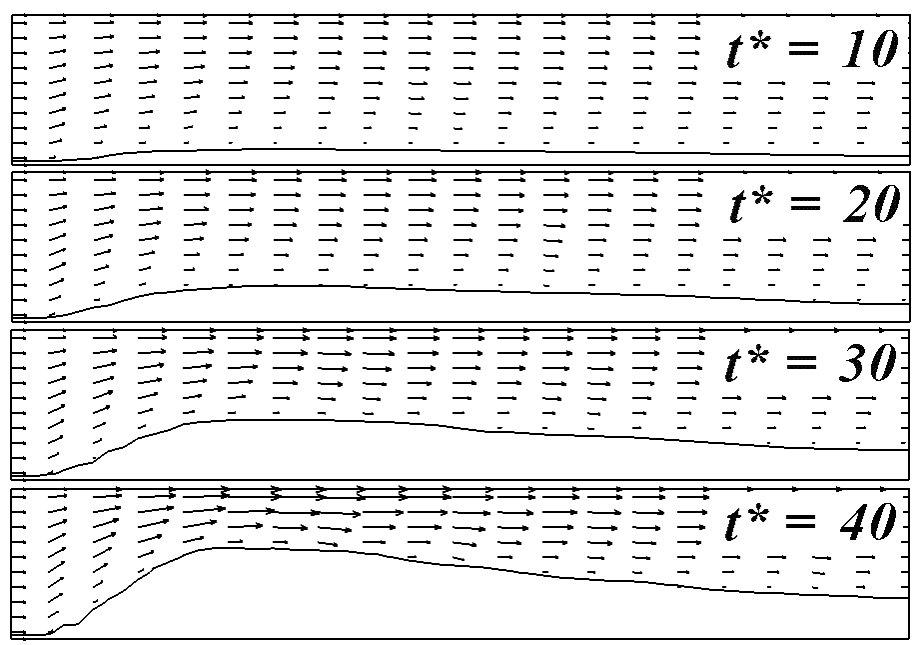

(c)

Figure 7: Evolution of the depositing front in a channel with flowing fluid with $\operatorname{Re}=1$ and $C_{o}^{*}=0.1$ for (a) $P e=15$ and $D a_{D}=10$, (b) $P e=15$ and $D a_{D}=5$ and (c) $P e=20$ and $D a_{D}=10$. 


\section{CONCLUDING REMARKS}

This article presents a general CFD framework for modeling of asphaltene deposition. The deposition front can be captured using either a level-set approach or a total concentration approach. As such a fixed mesh can be employed. The deposition process occurring at the depositing front is modeled as a first order reaction. Fluid flow is modeled using the incompressible Navier-Stokes equations. The presented approaches are validated against each other. It is then employed to study preliminarily the deposition process of asphaltene particles occurring in the flow of a two-dimensional channel.

\section{ACKNOWLEDGEMENT}

This work was supported by ADNOC Oil R\&D Subcommitee under Grant: 6000.1673.

\section{REFERENCES}

[1] D.L. Mitchell and J.G. Speight, The solubility of asphaltenes in hydrocarbon solvents, Fuel, vol. 52, 1973, pp. 149-152.

[2] J. Escobedo and G.A. Mansoori, Asphaltene and other heavyorganic particle deposition during transfer and production operations, SPE Annual Technical Conference and Exhibition, 1995, SPE 30672.

[3] M. Vafaie-Sefti, S.A. Mousavi-Dehghani and M. MohammadZadeh, A simple model for asphaltene deposition in petroleum mixtures, Fluid Phase Equilibria, vol. 206, 2003, pp. 1-11.

[4] R.A. Almehaideb, Asphaltene precipitation and deposition in the near wellbore region: a modeling approach, Journal of Petroleum Science and Engineering, vol. 42, 2004, pp. 157-170.

[5] A.R. Solaimany-Nazar and A. Zonnouri, Modeling of asphaltene deposition in oil reservoirs during primary oil recovery, Journal of Petroleum Science and Engineering, vol. 75, 2011, pp. 251259.

[6] E. Ramirez-Jaramillo, C. Lira-Galeana and O. Manero, Modeling asphaltene deposition in production pipelines, Energy \& Fuels, vol. 20, 2006, pp. 1184-1196.

[7] B.S. Soulgani, D. Rashtchian, B. Tohidi and M. Jamialahmadi, Integrated modeling methods for asphaltene deposition in wellstring, Journal of the Japan Petroleum Institute, vol. 52, 2009, pp. 322-331.

[8] D. Eskin, J. Ratulowski, K. Akbarzadeh and S. Pan, Modelling asphaltene deposition in turbulent pipeline flows, The Canadian Journal of Chemical and Engineering, vol. 89, 2011, pp. 421441.

[9] K. Akbarzadeh, D. Eskin, J. Ratulowski and S. Taylor, Asphaltene deposition measurement and modeling for flow assurance of tubings and flow lines, Energy \& Fuels, vol. 26, 2012, pp. 495-510.

[10] D. Eskin, J. Ratulowski, K. Akbarzadeh and S. Andersen, Modelling of asphaltene deposition in a production tubing, AIChE Journal, vol. x, 2011, pp. 1-13 (available online).

[11] F.M. Vargas, J.L. Creek and W.G. Chapman, On the Development of an Asphaltene Deposition Simulator, Energy \& Fuels, vol. 24, 2011, pp. 2294-2299.

[12] A.S. Kurup, F.M. Vargas, J. Wang, J. Buckley, J.L. Creek, Hariprasad, J. Subramani and W.G. Chapman, Development and application of an asphaltene deposition tool (ADEPT) for well bores, Energy \& Fuels, vol. 25, 2011, pp. 4506-4516.

[13] S. Osher and J.A. Sethian, Fronts propagating with curvaturedependent speed: Algorithms based on Hamilton-Jacobi formulations, Journal of Computational Physics, vol. 79, 1988, pp. 12-49.

[14] Ge Q., Yap Y.F., Vargas F.M., Zhang M. \& Chai J.C., A Total Concentration Method for Modeling of Deposition, Numerical Heat Transfer, Part B: Fundamentals, 2012, (accepted).

[15] S.V. Patankar, Numerical Heat Transfer and Fluid Flow, Hemisphere Publisher, New York, 1980.

[16] H.K. Versteeg and W. Malalasekera, An Introduction to Computational Fluid Dynamics: The Finite Volume Method, 2nd ed., Prentice Education Limited, England, 2007.

[17] G.-S. Jiang and D. Peng, Weighted ENO schemes for HamiltonJacobi equations, SIAM Journal of Scientific Computing, vol. 21, 2000, pp. 2126-2143.

[18] C.-W. Shu and S. Osher, Efficient Implementation of Essentially Non-Oscillatory Shock Capturing Schemes, Journal of Computational Physics, vol. 77, 1988, pp. 439-471.

[19] D. Peng, B. Merriman, S. Osher, H. Zhao and M. Kang, A PDEbased fast local level-set method, Journal of Computational Physics, vol. 155, 1999, pp. 410-438. 